\title{
Blood flow in S-shaped in-plane and out-of-plane coronary arteries $\begin{array}{ll}\text { P. R. Johnston } & \text { B. M. Johnston } \\ & 2\end{array}$
}

(Received 27 July 2007; revised 10 December 2007)

\begin{abstract}
The distribution of wall shear stress (WSS) in coronary arteries is an initiating factor for coronary artery disease. The complicated three dimensional structure of coronary arteries makes it difficult to isolate the factors affecting wss distributions. Here we present a computational fluid dynamics study of transient flow in simplified S-shaped arteries with in-plane and out-of-plane bends. For in-plane arteries there are two regions of consistently low wss during the cardiac cycle. The wss distribution is related to secondary velocities which appear as symmetric counter-rotating eddies and are approximately $8 \%$ of the axial velocity. For out-of-plane arteries the wss distributions are similar, but the eddies are no longer symmetric with secondary velocities only $7 \%$ of the axial velocities. The symmetric velocity patterns produced by the in-plane model could only exist in a single bend, which limits the usefulness of this model. The results obtained give useful insights into the nature of blood flow in realistically shaped coronary arteries.
\end{abstract}

See http://anziamj.austms.org.au/ojs/index.php/ANZIAMJ/article/view/330 for this article, (C) Austral. Mathematical Soc. 2008. Published January 15, 2008. ISSN 1446-8735 


\section{Contents}

\section{Introduction}

C342

2 The model

C344

2.1 Geometry ...................... C344

2.2 Governing equations . . . . . . . . . . . . . . C345

2.3 Computational mesh . . . . . . . . . . . . . . . C347

2.4 Parameters for comparison . . . . . . . . . . . . . C348

3 Results and discussion

C348

3.1 Wall shear stress . . . . . . . . . . . . . C348

3.2 Secondary velocities. . . . . . . . . . . . . . . C C351

3.3 Oscillatory shear index . . . . . . . . . . . . . C354

4 Conclusions

C355

References

C355

\section{Introduction}

Atherosclerotic lesions develop preferentially at certain sites in arteries such as the aortic arch, carotid bifurcation, infrarenal, femoral and coronary arteries $[1,4,5,10,16]$. Clinical observations found that plaques form predominantly on the inside of curves and the outer walls of vessel bifurcations $[1,7,10,16]$ in the arteries listed above and studies have shown that these sites co-localise with regions of low shear stress [8].

Several anatomical studies have shown that there is something different about plaque formation in coronary arteries compared to other arteries $[10,15]$. The main differences are that plaques form in both straight and curved sections of the arteries, which is not the case for other arteries. Rea- 
sons for such differences have been postulated as the complex pulsatile nature of the blood flow [14], the rheological properties of blood [3] and the geometry of the arteries, including branching, bifurcation and curvature $[7,8]$. Possible factors that may cause differences in the coronary arteries compared with other arteries are the particular character of the coronary inlet velocity waveform [11] and the fact that coronary velocities are lower than most of the other arteries mentioned [9, 11], which, with their smaller diameters, leads to lower Reynolds numbers [11]. Other factors may be their tortuous nature [1], which includes out-of-plane bends [2].

Although there are numerous studies of blood flow in various models of different human arteries, the starting point for this paper will be a recent study by Qiao et al. [13], who considered pulsatile flow in two in-plane Sshaped curves of 6 and $3 \mathrm{~mm}$ radii, respectively. The idea of their study was to numerically simulate blood flow in the aorta as well as other minor arteries. The study used an inlet velocity waveform corresponding to flow in the ascending aorta with a maximum velocity of $1.4 \mathrm{~m} / \mathrm{s}$ over a 0.4 second cycle, corresponding to a rather rapid pulse rate of 150 beats per minute.

This article presents simulations of blood flow in the coronary (small diameter) arteries. The aim is to use realistic coronary velocities up to $0.22 \mathrm{~m} / \mathrm{s}$ at a frequency of 60 beats per minute, a resting pulse rate. Two S-shaped coronary arteries are considered: firstly an in-plane artery (similar to that of Qiao et al. [13]) and secondly an artery with an out-of-plane bend. Simulations are presented for a left coronary artery using a velocity waveform measured at the entrance of the artery. In Section 3, comparisons are made between the in- and out-of-plane arteries, which are compared in terms of wall shear stress (WSS), secondary velocities and oscillatory shear index (OSI). 

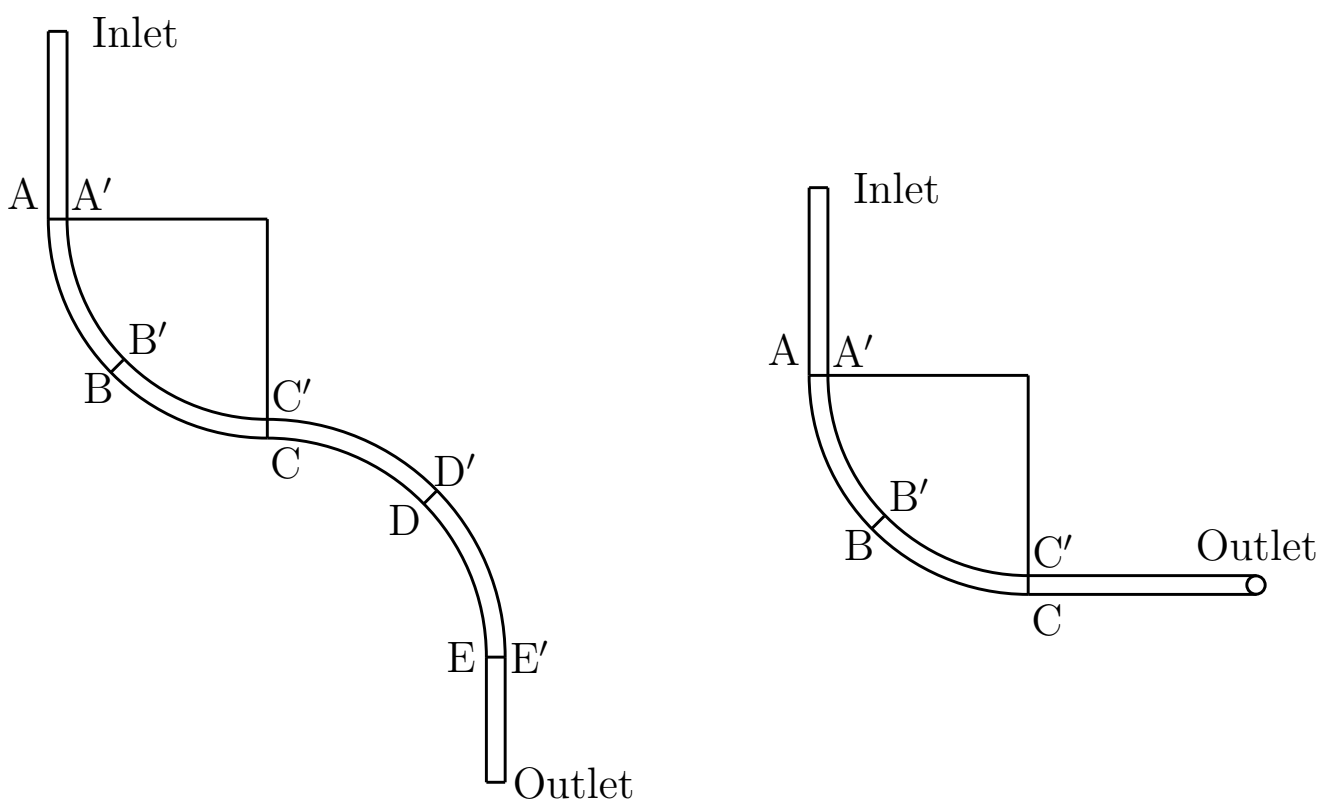

FiguRE 1: In-plane (left) and out-of-plane (right) artery models

\section{The model}

\section{$2.1 \quad$ Geometry}

Consider the in-plane S-shaped model shown in Figure 1(left). The straight inlet and outlet sections are of length $3 \mathrm{~cm}$ and $2 \mathrm{~cm}$, respectively, and the radius of curvature, $R_{c}$, of each curved section is $3.5 \mathrm{~cm}$, giving an overall artery length of $16 \mathrm{~cm}$. The cross-section of the artery model is circular with a radius of $2 \mathrm{~mm}$. Several particular cross-sections are indicated in the figure by the lines $A A^{\prime}, B B^{\prime}, C C^{\prime}, D D^{\prime}$ and $E E^{\prime}$ and these will be used to discuss results in Section 3.

To create the out-of-plane artery model, Figure 1(right), the second half 
of the artery, after the $C C^{\prime}$ plane, is rotated through $\pi / 2$ in the plane of $C C^{\prime}$ so the outlet now comes out of the page. The lines $D D^{\prime}$ and $E E^{\prime}$ are still present but not shown in Figure 1(right).

\subsection{Governing equations}

In these simulations blood is assumed to be incompressible fluid, which is governed by the Navier-Stokes equations

$$
\rho \frac{\partial \mathbf{v}}{\partial t}+\rho \mathbf{v} \cdot \nabla \mathbf{v}=\mu \nabla^{2} \mathbf{v}-\nabla P
$$

and the continuity equation

$$
\nabla \cdot \mathbf{v}=0
$$

where the velocity $\mathbf{v}$ is a three dimensional vector field, $P$ denotes pressure, $\rho$ denotes density $\left(1056 \mathrm{~kg} / \mathrm{m}^{3}\right)$ and $\mu$ denotes viscosity $(0.00345 \mathrm{Pas})$. This model assumes that blood is a Newtonian fluid, which has been demonstrated to be a reasonable assumption for the transient simulation of blood flow in arteries [6].

These equations are solved subject to the following boundary conditions.

1. The artery walls are assumed rigid (which is a common assumption [3, $12,13,14]$ used to ease computational load), upon which a no-slip condition is imposed on $\mathbf{v}$.

2. At the outlet, the velocities are free but the normal and tangential stresses are constrained to be zero and the gauge pressure is set to zero.

3. At the inlet, a uniform velocity profile is used with a time varying forcing function representative of flow in the left coronary artery, Figure 2 [12]. The forcing function is scaled to have a maximum inlet velocity $v_{\max }=0.214 \mathrm{~m} / \mathrm{s}$ with a period $T=1$ second $(60$ heart beats per minute). 


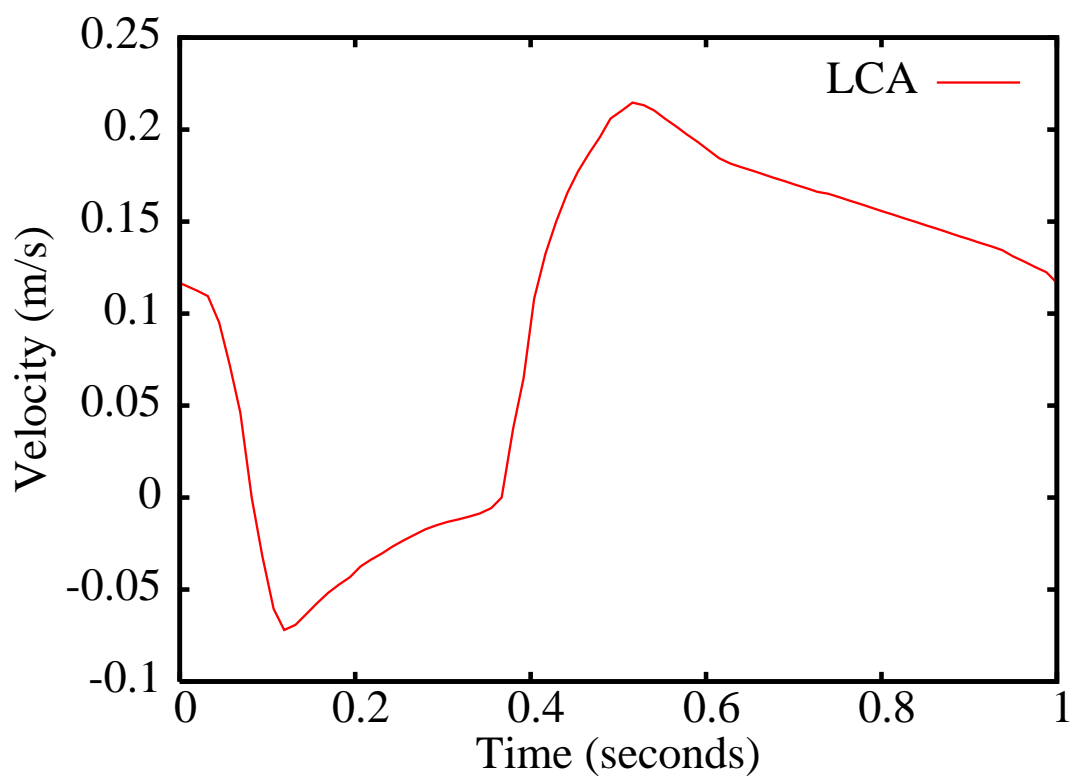

FiguRE 2: Waveform of the velocity forcing function for a left coronary artery. 
To compare this model with other flow studies, the following dimensionless parameters are used: the Reynolds number, Re; the Womersley number, $\alpha$, and the Dean number, Dn. For the simulations presented here, the parameters are defined as and evaluate to:

1. Peak Reynolds number

$$
\mathrm{Re}=\frac{2 \rho R V_{\max }}{\mu}=226,
$$

2. Womersley parameter

$$
\alpha=\sqrt{\frac{2 \pi \rho}{T \mu}} R=0.057,
$$

3. Dean Number

$$
\operatorname{Dn}=\operatorname{Re} \sqrt{\frac{R}{R_{c}}}=54 .
$$

\subsection{Computational mesh}

The nonlinear governing equations were solved using the CFD-ACE suite of packages (CFDRC, Huntsville, Alabama, USA). The solution procedure utilises the finite volume method and is based on the SIMPLE family of algorithms. Meshes were created using the GEOM package of the CFD-ACE suite. The inlet mesh is a typical butterfly mesh with 18 nodes along each side of the centre square and 25 nodes along the 'wings' of the mesh. Nodes are clustered towards the walls of the artery to improve the calculation of the wall shear stress. The volume mesh is created by extruding the inlet mesh along the centreline of the artery employing a total of 96 nodes over the length of the artery, giving a total of 192,882 nodes for the mesh. This figure was determined by increasing the number of nodes until changes in the oscillatory shear index were less than $0.1 \%$ over the entire artery wall. 


\subsection{Parameters for comparison}

To study the differences between the in-plane and out-of-plane arteries the following comparisons will be used:

1. The wall shear stress (wss)

$$
\tau_{w}=-\left.\mu \frac{\partial \mathbf{v}}{\partial n}\right|_{\text {wall }},
$$

which is related to the outward normal derivative of the velocity at the wall.

2. Secondary velocities in the planes $A A^{\prime}, B B^{\prime}, C C^{\prime}, D D^{\prime}$ and $E E^{\prime}$ along the artery.

3. The oscillatory shear index

$$
\mathrm{OSI}=\frac{1}{2}\left[1-\frac{\left|\int_{0}^{T} \tau_{w} d t\right|}{\int_{0}^{T}\left|\tau_{w}\right| d t}\right],
$$

which is a form of time averaged wall shear stress over one heart beat.

\section{Results and discussion}

\subsection{Wall shear stress}

During the majority of the cardiac cycle there is forward flow (Figure 2) and the wsS is qualitatively similar to that shown in Figure 3, with high wss on the outside of curves and low wss on the inside of curves. Figure 3(left) shows 

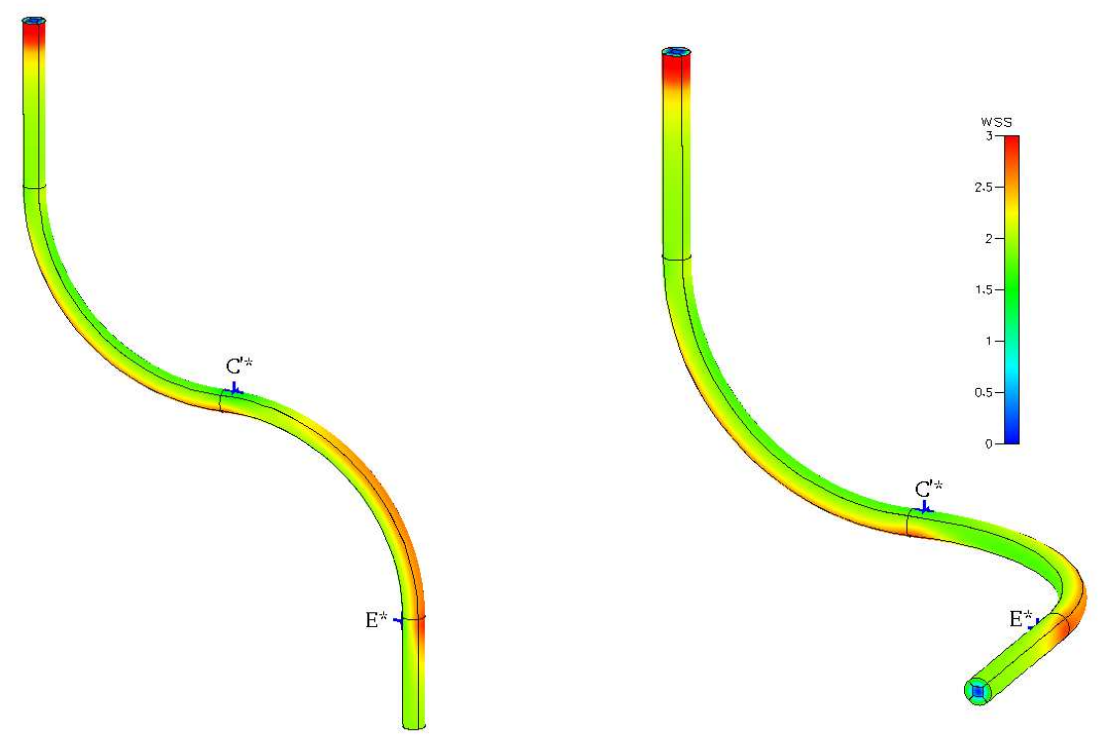

FiguRE 3: Wall shear stress distributions at peak flow in the in-plane (left) and out-of-plane (right) artery models. 


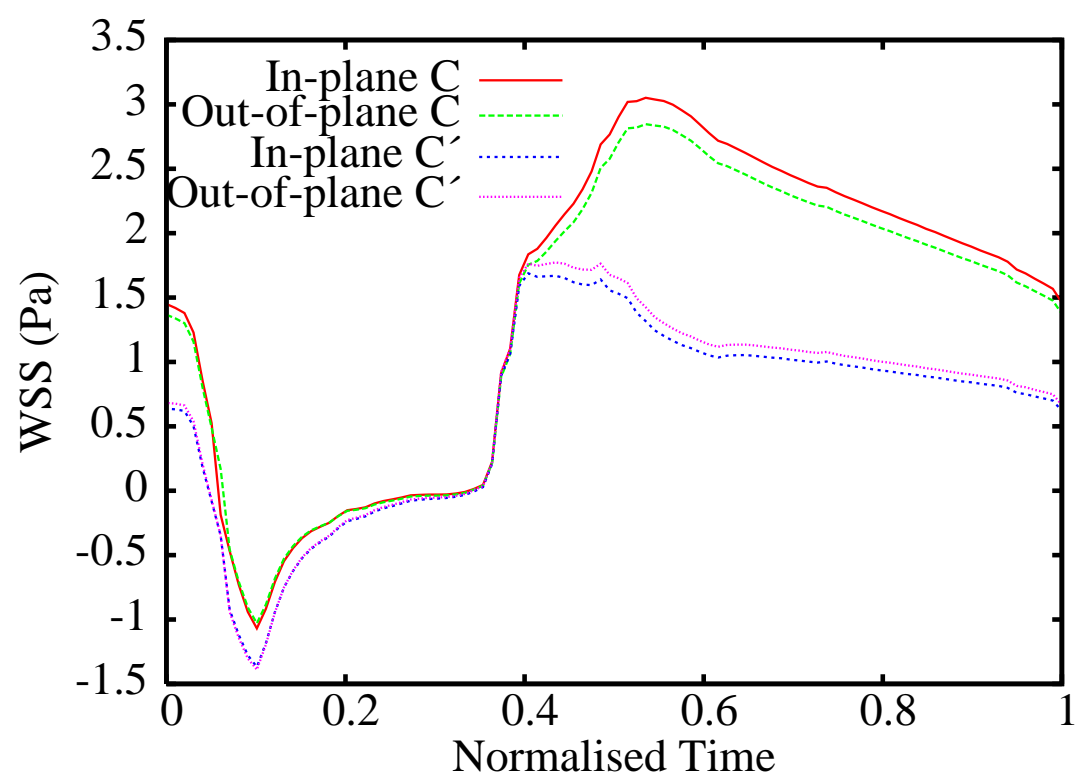

Figure 4: Trace of wss at the points $C$ and $C^{\prime}$ throughout the heart beat cycle.

WSS for in-plane artery model at (normalised) time $t=0.52$, which corresponds to peak forward flow for that artery. Interestingly, the pattern of wsS is the same for the out-of-plane model (Figure 3(right)) but the wss distribution wraps smoothly around the artery between the planes $C C^{\prime}$ and $D D^{\prime}$. At other times of forward flow, the pattern of wss is very similar to those shown in Figure 3, but the magnitudes vary with the velocity waveform (Figure 2).

Figure 4 shows that wss at $C$ is always greater than or equal to wss at $C^{\prime}$. This is consistent with the wss plots in Figure 3, which show spots of high wss at $C$ and low wss at $C^{\prime}$, for both artery models, at the particular 
instant in time $(t=0.52)$ chosen. However, the magnitude of the negative wss is greater at $C^{\prime}$ than at $C$ and so, for the portion of the cycle where there is back-flow (Figure 2, $t=0.08$ to $t=0.37$ ), wss is 'larger' on the inside rather than the outside of the curve, since negative Wss indicates reversed direction. Another feature of Figure 4 is that WSS is very similar in both models and this is in agreement with the similarities between in the distributions in Figure 3. In addition, similar graphs (not presented) at $E$ and $E^{\prime}$ show wss which is almost the same as at $C^{\prime}$ and $C$, respectively, where the dash and non-dash reversal occurs because $E$ is now on the inside of the bend and therefore corresponds to $C^{\prime}$, and so on.

\subsection{Secondary velocities}

Examination of secondary velocities in the planes $B B^{\prime}, C C^{\prime}, D D^{\prime}$ and $E E^{\prime}$ indicates differences in flow patterns between the two artery models. For the majority of the cycle the flow profiles show two eddies, one in each of the top and bottom halves of the artery, with flow in these in opposite directions. A series of these plots was constructed for various time intervals and the characteristics of these plots are summarised in Table 1 . The entries in this table are of the form $\# \$^{*}$ where $\#=$ number of eddies, $\$=$ rotation direction of the topmost eddy ( $\mathrm{c}=$ clockwise, $\mathrm{a}=$ anti-clockwise) and $*=$ symmetry of eddies about the plane of curvature ( $\mathrm{s}=$ symmetric, $\mathrm{n}=$ not symmetric).

Four key features observed from Table 1 are the following

1. During forward flow, two eddies are always seen, while during back flow two eddies are seen at $B B^{\prime}$ and $D D^{\prime}$, but never at $E E^{\prime}$ and only once at $C C^{\prime}$.

2. At $B B^{\prime}$ and $C C^{\prime}$, the top eddy rotates in a clockwise direction (except at $C C^{\prime}$ during back flow), whereas at $D D^{\prime}$ and $E E^{\prime}$, the rotation direction of the top eddy is anti-clockwise. 
TABLE 1: Summary of secondary velocity flow patterns at various points of the in-plane (IP) and out-of-plane (OoP) artery models (c=clockwise, a=anticlockwise, $\mathrm{s}=$ symmetric, see text for full explanation of the notation).

\begin{tabular}{|c|cccccccc|}
\hline Time & IP & OoP & IP & OoP & IP & OoP & IP & OoP \\
Period & $B B^{\prime}$ & $B B^{\prime}$ & $C C^{\prime}$ & $C C^{\prime}$ & $D D^{\prime}$ & $D D^{\prime}$ & $E E^{\prime}$ & $E E^{\prime}$ \\
\hline $0.01-0.10$ & $2 \mathrm{cs}$ & $2 \mathrm{cs}$ & $2 \mathrm{cs}$ & $2 \mathrm{cs}$ & 2 as & $2 \mathrm{an}$ & $2 \mathrm{as}$ & $2 \mathrm{as}$ \\
$0.11-0.19$ & $2 \mathrm{cs}$ & $2 \mathrm{cs}$ & 0 & 0 & $2 \mathrm{as}$ & $2 \mathrm{an}$ & 0 & 0 \\
$0.20-0.37$ & $2 \mathrm{cs}$ & $2 \mathrm{cs}$ & $2 \mathrm{as}$ & $2 \mathrm{an}$ & $2 \mathrm{as}$ & $2 \mathrm{as}$ & 0 & 0 \\
$0.38-0.41$ & $2 \mathrm{cs}$ & $2 \mathrm{cs}$ & 0 & 0 & $2 \mathrm{as}$ & $2 \mathrm{as}$ & 0 & 0 \\
$0.42-1.00$ & $2 \mathrm{cs}$ & $2 \mathrm{cs}$ & $2 \mathrm{cs}$ & $2 \mathrm{cs}$ & 2as & 2as & 2as & $2 \mathrm{as}$ \\
\hline
\end{tabular}

3. In the in-plane artery model, the eddies are symmetric with respect to the plane of curvature.

4. In the out-of-plane artery model, the eddies are symmetric at $B B^{\prime}$, $C C^{\prime}$ and $E E^{\prime}$; at $D D^{\prime}$, they are often not symmetric.

The symmetric flow patterns produced by the in-plane artery model reflects a limitation of that model. For this case it would be almost possible to predict the flow patterns by considering a series of single bends in the artery. However, since coronary arteries sit on the curved surface of the heart, no symmetry plane can exist and the in-plane model is of limited use.

The ratio of mean secondary velocity to mean axial velocity in the $C C^{\prime}$ plane for the in-plane model was $8.2 \%$ and for the out-of-plane model it was $7.3 \%$. In the $E E^{\prime}$ plane the ratio was $6.3 \%$ for both models. 

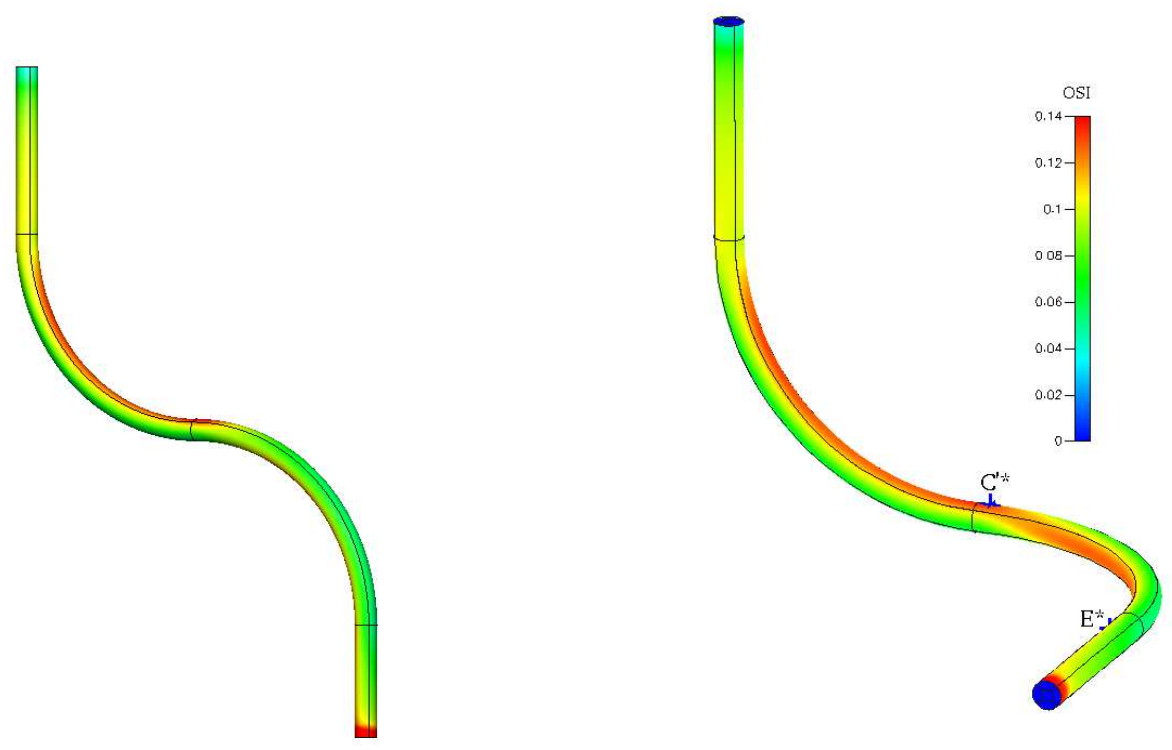

Figure 5: Oscillatory shear index in the in-plane (left) and out-of-plane (right) artery models. 
TABLE 2: Values of mmWss and Osi at the points of mmwss and next mmWss for the in-plane (IP) and out-of-plane (OoP) artery models

\begin{tabular}{ccccccc}
\hline $\begin{array}{c}\text { Artery } \\
\text { Model }\end{array}$ & $\begin{array}{c}\text { Time of } \\
\text { mmwss }\end{array}$ & $\begin{array}{c}\text { Value of } \\
\text { mmwss }\end{array}$ & $\begin{array}{c}\text { OsI at } \\
\text { mmwss }\end{array}$ & $\begin{array}{c}\text { Time of } \\
\text { next } \\
\text { mmWss }\end{array}$ & $\begin{array}{c}\text { Value of } \\
\text { next } \\
\text { mmWss }\end{array}$ & $\begin{array}{c}\text { OsI at } \\
\text { next } \\
\text { mmWSS }\end{array}$ \\
\hline IP & 0.44 & 1.61 & 0.14 & 0.44 & 1.70 & 0.14 \\
OoP & 0.44 & 1.71 & 0.13 & 0.44 & 1.73 & 0.14 \\
\hline
\end{tabular}

\subsection{Oscillatory shear index}

A plot of OsI for both artery models is shown in Figure 5 and when this is compared with Figure 3, which shows wss for the same arteries, it is apparent that positions of low wsS are positions of high OSI and vice versa. For the inplane model (Figure 5(left)) there is an abrupt change in OsI just downstream of the $C C^{\prime}$ plane. However, for the out-of-plane model (Figure 5(right)) there is a gradual change in OSI wrapping around the artery in a helical fashion from $C^{\prime}$ to $E$.

To examine the relationship between extremes in wall shear stress and extremes in OSI, positions of the lowest maximum wsS at any point over the heart beat cycle were calculated and denoted by mmwss. These points, denoted $C^{*}$ and $E^{*}$ (see Figure 3 ), are the same for both arteries and are very close to the points $C^{\prime}$ and $E$. At these points the OSI was also determined and the results are given in Table 2. In general, these values of osI are very near to maximum values. Table 2 shows that low values of wss correspond to high values of OSI, as discussed above. The OSI in the in-plane model is a little higher than in out-of-plane model at the same point. 


\section{Conclusions}

Simulations have been presented for blood flow in coronary arteries of simplified geometry. In-plane and out-of-plane models were used in order to avoid the complex nature of realistic coronary arteries. Simulations show that differences exist between flow parameters (Wss, secondary velocities, OSI) in these two simplified models. The in-plane model is too much of a simplification for considering multiple bends in an artery. It produces a symmetric flow pattern which could only exist in a single bend. The out-of-plane model allows for a smooth transition region between two bends in the artery.

In order to achieve useful insights into blood flow in realistic coronary arteries using simplified models it is necessary to at least consider flow with some form of out-of-plane bend. The degree of out-of-plane nature of the model does not need to be the relatively extreme $\pi / 2$ rotation used here. Useful insights would be obtained by using less extreme degrees of rotation and flow through bends of less than $90^{\circ}$.

\section{References}

[1] Toshihisa Asakura and Takeshi Karino. Flow patterns and spatial distribution of atherosclerotic lesions in human coronary arteries. Circ. Res., 66:1054-1066, 1990. C342, C343

[2] C. G. Caro, D. J. Doorley, M. Tarnawski, K. T. Scott, Q. Long, and C. L. Dumoulin. Non-planar curvature and branching of arteries and non-planar-type flow. Proc. R. Soc. Lond. A, 452:185-197, 1996. C343

[3] Jie Chen and Xi-Yun Lu. Numerical investigation of the non-newtonian pulsatile blood flow in a bifurcation model with a non-planar branch. Journal of Biomechanics, 39(5):818-832, 2006. doi:10.1016/j.jbiomech.2005.02.003 C343, C345 
[4] M. H. Friedman, O. J. Deters, F. F. Mark, C. B. Bargeron, and G. M. Hutchins. Aterial geometry affects hemodynamics: A potential risk factor for athersclerosis. Atherosclerosis, 46:225-231, 1983. doi:10.1016/0021-9150(83)90113-2 C342

[5] H. W. Hoogstraten, J. G. Kootstra, B. Hillen, J. K. B. Krijger, and P. J. W. Wensing. Numerical simulation of blood flow in an artery with two successive bends. Journal of Biomechanics, 29:1075-1083, 1996. doi:10.1016/0021-9290(95)00174-3 C342

[6] B. M. Johnston, P. R. Johnston, S. Corney, and D. Kilpatrick.

Non-Newtonian blood flow in human right coronary arteries: Transient simulations. Journal of Biomechanics, 39(6):1116-1128, 2006. doi:10.1016/j.jbiomech.2005.01.034 C345

[7] D. Liepsch. An introduction to biofluid mechanics - basic models and applications. Journal of Biomechanics, 35:415-435, 2002. doi:10.1016/S0021-9290(01)00185-3 C342, C343

[8] A. M. Malek, L. A. Seth, and S. Izumo. Hemodynamic shear stress and its role in atherosclerosis. JAMA, 282(21):2035-2042, 1999. C342, C343

[9] R. M. Nerem, J. A. Rumberger, D. R. Gross, W. W. Muir, and G. L. Gelger. Hot-film coronary artery velocity measurements in horses. Cardiovasc. Res., 10:301-313, 1976. C343

[10] M. Ojha, R. L. Leask, J. Butany, and K. W. Johnston. Distribution of intimal and medial thickening in the human right coronary artery: a study of 17 RCAs. Atherosclerosis, 158:147-153, 2001. doi:10.1016/S0021-9150(00)00759-0 C342

[11] T. J. Pedley. The fluid mechanics of large blood vessels. Cambridge University Press, 1980. C343 
[12] K. Perktold, R. M. Nerem, and R. O. Peter. A numerical calculation of flow in a curved tube model of the left main coronary artery. J. Biomechanics, 24(3-4):175-189, 1991. doi:10.1016/0021-9290(91)90176-N C345

[13] A. K. Qiao, X. L. Guo, S. G. Wu, Y. J. Zeng, and X. H. Xu. Numerical study of nonlinear pulsatile flow in S-shaped curved arteries. Medical Engineering and Physics, 26:545-552, 2004. doi:10.1016/j.medengphy.2004.04.008 C343, C345

[14] D. A. Steinman, D. A. Vorp, and C. R. Ethier. Computational modeling of arterial biomechanics: Insights into pathogenesis and treatment of vascular disease. Journal of Vascular Surgery, 37(5):1119-1127, 2003. doi:10.1067/mva.2003.122 C343, C345

[15] J. C. Wang, S.-L. T. Normand, L Mauri, and R. E. Kuntz. Coronary artery spatial distribution of acute myocardial infarction occlusions. Circulation, 110:278-284, 2004. doi:10.1161/01.CIR.0000135468.67850.F4 C342

[16] C. K. Zarins, D. P. Giddens, B. K. Bharadvaj, V. S. Sottiurai, R. F. Mabon, and S. Glagov. Carotid bifurcation atherosclerosis: quantitative correlation of plaque localization with flow velocity profiles and wall shear stress. Circ. Res., 53:502-514, 1983. C342 


\section{Author addresses}

1. P. R. Johnston, School of Biomolecular and Physical Sciences Griffith University, Nathan, Australia. mailto:p.johnston@griffith.edu.au

2. B. M. Johnston, School of Biomolecular and Physical Sciences Griffith University, Nathan, Australia. 